\title{
What Really Caused the ROKS Cheonan Warship Sinking?
}

\author{
Hwang Su Kim ${ }^{1}$ and Mauro Caresta ${ }^{2}$ \\ ${ }^{1}$ Department of Physics, Kyungsung University, Pusan 608-736, Republic of Korea \\ ${ }^{2}$ Department of Engineering, University of Cambridge, Cambridge CB2 1PZ, UK
}

Correspondence should be addressed to Hwang Su Kim; jwa@ks.ac.kr

Received 25 May 2014; Revised 8 August 2014; Accepted 27 August 2014; Published 20 November 2014

Academic Editor: Emil Manoach

Copyright (C) 2014 H. S. Kim and M. Caresta. This is an open access article distributed under the Creative Commons Attribution License, which permits unrestricted use, distribution, and reproduction in any medium, provided the original work is properly cited.

\begin{abstract}
This paper is concerned with the sinking of the Korean naval warship (ROKS Cheonan) and the reported spectra of the seismic signals recorded at the time of the incident. The spectra of seismic signals show prominently amplitude peaks at around $8.5 \mathrm{~Hz}$ and its harmonics. These frequencies were explained with the vibrations of a water column due to an underwater explosion. This explanation is highly doubtful and concerns about its validity have already been raised in the scientific community. In this work an alternative explanation is presented: it is shown that the recorded seismic spectra are consistent with the natural frequencies of vibrations of a large submarine with a length of around $113 \mathrm{~m}$. This finding raises the possibility that the ROKS Cheonan sunk because of the collision with a large submarine rather than the explosion of a torpedo or an underwater mine.
\end{abstract}

\section{Introduction}

Several years have passed since the incident of the Korean naval warship (ROKS Cheonan) sinking that occurred on the 26th of March $2010[1,2]$. The Cheonan warship was split largely into two parts as shown in Figure 1 and sank off Korea's west coast near Baekryeong Island in the Yellow Sea. The seismic signals were recorded at the time of the incident in many stations including Baekryeong Island. The analysis of the seismic signals followed soon after the incident as reported in [3]. Based on the official government report of a summary of its investigation on 20 May 2010, the warship was sunk by a North Korean torpedo fired by a midget submarine (JIG's report [1] printed in September 2010). Nevertheless controversy over the scientific legitimacy of the report has been aroused among scientists [4-8]. The doubts raised by each of the reference are summarized in Appendix A.

This work was motivated from the report of $[3,9]$ showing the spectra of the seismic signals generated from the Cheonan incident: the spectral Figure 7 in [3] shows very interesting spectral peaks at around $8.5 \mathrm{~Hz}$ and its harmonics. The author in [3] has explained these characteristic frequencies as those of the hydroacoustic reverberation waves generated by an underwater explosion. This explanation is based on the theory in the report of [10] that underwater explosion generates reverberation waves in the column of seawater with characteristic odd harmonic series of a fundamental frequency by $f_{1}=c / 4 \mathrm{H}$, where $c$ is the wave speed in water $(\sim 1.5 \mathrm{~km} / \mathrm{s})$ and $\mathrm{H}$ is the water column depth (for $f_{1}=8.5 \mathrm{~Hz}$, $\mathrm{H}=44 \mathrm{~m}$ ). However contrary to this prediction, Figure 7 in [3] clearly shows a series including $2 f_{0} \approx 17.7 \mathrm{~Hz}$ and $4 f_{0} \approx 34.4 \mathrm{~Hz}$, which is not an odd harmonic series. Thus it is very questionable for the validity of the analysis result in [3]. Recently in another paper based on the same underwater explosion theory as in [9] where the explosive source was indicated as a South Korean land control mine rather than a North Korean torpedo this problem was recognized and the authors stated that "these unusual unexplained spectral peaks at $17 \mathrm{~Hz}$ and its second harmonic frequency at $34 \mathrm{~Hz}$ can be attributed to the nonlinear deformation of the hull structure by shock waves along with overlapping readings from later arriving T-phases."

This statement in our opinion is a speculation and no proof or simulations to support it were shown in [9]; besides the corresponding amplitude matching of the recorded spectra had not been attempted in $[3,9]$.

We think that it is worthwhile to search an alternative theory to explain more properly the spectra including amplitudes of the seismic signals. For instance, it will be shown in this work that a possible explanation for the sinking 


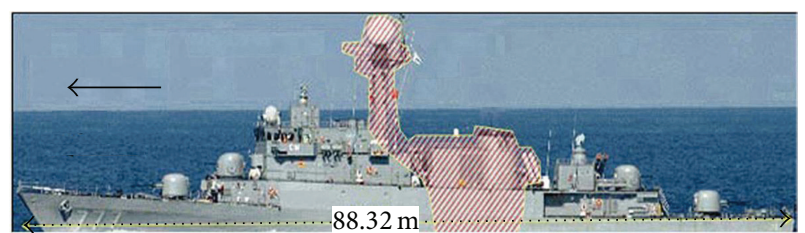

(a)

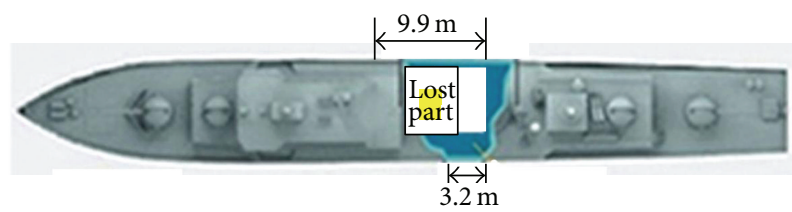

(b)

FIGURE 1: (a) ROKS warship; (b) top view. Sketch of the part damaged during the incident.

of the ROKS Cheonan warship is a collision with a large submarine. The reason for this explanation relies on the fact the frequencies observed in the spectra of the seismic data are consistent with the natural frequencies of vibrations of a large submarine, as it will be shown later in the paper. The collision of the ship with the submarine would generate a large force transmitted to the submarine hull with the consequent structure born sound radiated in the sea-water. The sound waves then transmit into the earth crust as seismic waves and can be recorded at seismic detecting stations. The purpose of this paper is to explore this possibility to gain insight into the reality of the cause of the Cheonan sinking. For this aim both a simplified and more accurate model of a submarine hull will be presented in the following sections. A simplified model will be used first to find the main dimension of the submarine hull; a more realistic model will be then used to properly match the seismic signature.

\section{Theoretical Formulation and Calculation}

2.1. Natural Vibrations of a Submarine Hull. A model of a submarine is presented according to the approach of [11, 12 ], where the submarine was modeled as having a main cylindrical hull with internal bulkheads and ring stiffeners. The cylindrical shell is closed by truncated conical shells, which are closed by circular plates at each end as shown in Figure 2(a). Hereafter we call this the realistic model of a submarine; however we also consider a simplified version, as shown in Figure 2(b), where the hull is modeled as a cylindrical shell with ring stiffeners closed by circular plates at ends of the shell. This is because the effect of the end cones on the structural response is minimal [11] and the purpose of using the simplified model is to get a closed form solution for the main parameters of the hull and get a first matching with the recorded seismic frequencies. The detailed model as in [12] will be used later to calculate a more realistic structural and acoustic response of the submarine.

At first, let us consider a cylindrical shell of length $L$ with shear diaphragm boundary conditions to model the

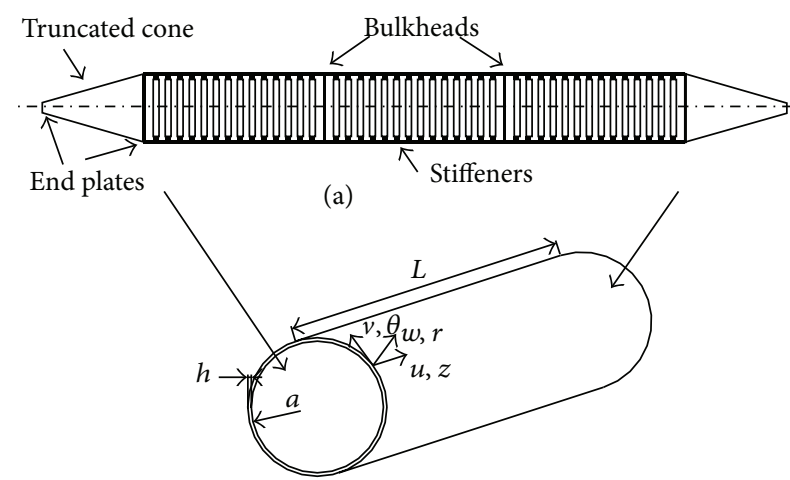

(b)

FIGURE 2: (a) Schematic diagram of the more realistic model of a submarine for the free-free cylindrical shell with bulkheads, stiffeners, and closed by plates following conical end caps. (b) Cylindrical coordinate system $(z, \theta$, and $r)$ and the corresponding displacements $(u, v$, and $w)$ for a thin walled shell. The figure indicates the simplified model of a submarine for the cylindrical shell, stiffeners, and closed by plates at ends of the shell.

main part of the hull (Figure 2(b)). The equations of motion and the solutions and the boundary condition equation are given in Appendix B. The solution of the free vibration of the cylindrical shell satisfying the Flügge differential equations of motion can be written as given by Caresta et al. [12, 13]:

$$
\begin{gathered}
u^{(n)}(z, \theta, t)=U^{(n)} \cos (n \theta) \cos (k z) e^{-j w t}, \\
v^{(n)}(z, \theta, t)=V^{(n)} \sin (n \theta) \sin (k z) e^{-j \omega t}, \\
w^{(n)}(z, \theta, t)=W^{(n)} \cos (n \theta) \sin (k z) e^{-j w t} .
\end{gathered}
$$

Here $u^{(n)}, v^{(n)}$, and $w^{(n)}$ are the orthogonal components of shell displacements in the $z$-axial, the $\theta$-circumferential, and the $r$-radial directions, respectively. $n$ is the circumferential mode number $(n=0,1,2 \ldots), j$ is the imaginary unit, $\omega$ is the angular frequency, and $k$ is the axial wave number. The shear diaphragm boundary conditions are given by

$$
\begin{array}{r}
v^{(n)}(z, \theta, t)=0, \\
w^{(n)}(z, \theta, t)=0, \\
N_{z}=M_{z}=0, \\
z=0, L,
\end{array}
$$

where $N_{z}$ is the membrane force and the $M_{z}$ is the bending moment. With these boundary conditions, the axial wave number $k$ is given by

$$
k_{m}=\frac{m \pi}{L}, \quad m=0,1,2 \ldots
$$

Substituting (1) and (3) into the Flügge equations gives the eigenvalue equation in the matrix form

$$
\left[\begin{array}{ccc}
L_{11}-\Omega^{2} & L_{12} & L_{13} \\
L_{21} & L_{22}-\Omega^{2} & L_{23} \\
L_{31} & L_{32} & L_{33}-\Omega^{2}
\end{array}\right]\left[\begin{array}{c}
U^{(n)} \\
V^{(n)} \\
W^{(n)}
\end{array}\right]=0
$$


In (4) $\Omega$ is the dimensionless frequency parameter given by $\Omega=\omega \sqrt{\gamma} a / c_{L}$. The elements of the matrix are given by

$$
\begin{gathered}
L_{11}=(k a)^{2}+\frac{(1-v)}{2} n^{2}\left(1+\beta^{2}\right) \\
L_{12}=-\frac{(1+v)}{2} n k a \\
L_{13}=-v k a-\beta^{2}\left\{(k a)^{3}-\frac{(1-v)}{2} n^{2} k a\right\} \\
L_{21}=L_{12} \\
L_{22}=\frac{(1-v)}{2}(k a)^{2}\left(1+3 \beta^{2}\right)+n^{2}(1+\mu) \\
L_{31}=L_{13}(1+\mu+\chi)-\chi n^{3}+\beta^{2} \frac{(3-v)}{2} n(k a)^{2} \\
L_{32}=L_{23} \\
L_{33}=(1+\mu+2 \chi)-2 \chi n^{2}+\beta^{2}\left\{\left[(k a)^{2}+n^{2}\right]^{2}+1-2 n^{2}\right\}
\end{gathered}
$$

where

$$
\begin{gathered}
\Omega^{2}=\frac{\gamma a^{2}}{c_{L}^{2}} \omega^{2}, \quad \gamma=\left(1+\frac{A}{h b}+\frac{m_{\mathrm{eq}}}{\rho h}\right), \\
c_{L}^{2}=\frac{E}{\rho\left(1-v^{2}\right)}, \quad \beta^{2}=\frac{h^{2}}{12 a^{2}} . \\
\mu=\frac{\left(1-v^{2}\right) A}{h b}, \quad \chi=\frac{\left(1-v^{2}\right) A z_{e}}{h b a} .
\end{gathered}
$$

In the previous equations, $a$ is the mean radius of the cylindrical shell, $h$ is the shell thickness, $A$ is the cross-section area of the ring stiffener, $b$ is the stiffener spacing, and $z_{e}$ is the distance between the shell mid surface and the center of a ring. $E, \rho$, and $v$ are Yong's modulus, density, and Poisson's ratio of the shell, respectively. $c_{L}$ is the longitudinal wave speed in the shell and $m_{\mathrm{eq}}$ is the equivalent distributed mass on the shell to take into account the onboard equipment and ballast tanks. For the each set of $(n, m)$ the matrix equation of (4) gives three real eigenvalues of $\Omega^{2}$ and the corresponding eigenvectors $\left(U^{(n)}, V^{(n)}\right.$, and $\left.W^{(n)}\right)$ under the usual normalization condition $\left|U^{(n)}\right|^{2}+\left|V^{(n)}\right|^{2}+\left|W^{(n)}\right|^{2}=1$.

2.2. Axisymmetric Modes of Vibrations. If $n=0$, the wave modes are independent of $\theta$ and if we do not consider the torsional modes, we can set $v^{(n)}(z, \theta, t)=0$. The matrix equation (4) simplifies in a $2 \times 2$ matrix equation:

$$
\left[\begin{array}{cc}
L_{11}-\Omega^{2} & L_{12} \\
L_{21} & L_{22}-\Omega^{2}
\end{array}\right]\left[\begin{array}{c}
U^{(n)} \\
W^{(n)}
\end{array}\right]=0
$$

where

$$
\begin{gathered}
L_{11}=(k a)^{2}, \quad L_{12}=-v k a-\beta^{2}(k a)^{3} \\
L_{21}=L_{12}, \quad L_{22}=(1+\mu+2 \chi)+\beta^{2}\left\{(k a)^{4}+1\right\} .
\end{gathered}
$$

Under the condition, $(k a)<1$, the off-diagonal elements become small values and then the solutions of (6a) approximately give for the nonzero of $\left(U^{(n)}, V^{(n)}\right)$

$$
L_{11}-\Omega^{2} \approx 0, \quad L_{22}-\Omega^{2} \approx 0 .
$$

The first equation (7) with (3) and the first relation in (5j) and $L_{11}=(k a)^{2}$ in $(6 \mathrm{~b})$ give

$$
f_{m}^{(0)} \approx \frac{m}{2 L} \frac{c_{L}}{\sqrt{\gamma}}, \quad \text { where } m=1,2 \ldots
$$

It is very important to note that (8) is a harmonic series with a fundamental frequency of $f_{1}^{(0)}$, which can be used to match the characteristic frequencies of the seismic signals reported in $[3,9]$. The validity of $(8)$ in the approximation can be seen up to the wave number $m=8$ from the solution of (6a). The first 4 frequencies and the corresponding normalized eigenvectors of (6a) are listed in Table 1. Using (8) and (5j), we can obtain analytically the length $L$ of a submarine giving $f_{1}^{(0)}=8.5 \mathrm{~Hz}$ as to match the recorded seismic signature. The submarine hull is assumed to be made of steel with physical properties of $\rho \approx 7800 \mathrm{~kg} / \mathrm{m}^{3}, E \approx 2.1 \times 10^{11} \mathrm{~N} / \mathrm{m}^{2}$, and $v \approx 0.3 ; c_{L}=5.450 \mathrm{~km} / \mathrm{s}$. It is widely known that the typical dimension of submarines has the ratio of $2 a / L=\sim 0.1$ and $h / a=\sim 0.01$. In $\gamma=\left(1+A / h b+m_{\mathrm{eq}} / \rho h\right), A=0.08 \mathrm{~m} \times$ $0.15 \mathrm{~m}$ and $b=0.5 \mathrm{~m}$ can be used as in [12]. The stiffeners parameters $A$ and $b$ give minor contribution in $\gamma$ so it is not necessary to know accurate values. $m_{\mathrm{eq}}$ is supposed to be less than the value estimated by the relation $m_{\mathrm{eq}}=\rho_{f} V_{s} / S_{s}$ where $V_{s}$ and $S_{s}$ are the volume and the surface area of submarine, respectively. $\rho_{f}$ is the density of seawater, about $1025 \mathrm{~kg} / \mathrm{m}^{3}$. If $V_{s}=\pi a^{2} L$ and $S_{s}=2 \pi a L, m_{\mathrm{eq}}=\rho_{f} a / 2$. Inserting these values in (8) gives $L=113 \mathrm{~m}$. This value is the typical length of a large submarine. In summary we have $L=113 \mathrm{~m}, a=5.65 \mathrm{~m}$ (radius of the cylindrical model), $h=0.0565 \mathrm{~m}$ (thickness of the hull), and $m_{\mathrm{eq}}=$ $2896 \mathrm{~kg} / \mathrm{m}^{2}$ giving $\sqrt{ } \gamma=2.84$. This model of submarine according to (8) has the natural frequencies as a harmonic series of $8.5 \mathrm{~Hz}$. In this result the equivalent mass is found to be $m_{\mathrm{eq}}=2896 \mathrm{~kg} / \mathrm{m}^{2}$ and it seems a little too high. For a submarine with a length of $113 \mathrm{~m}$, considering about 400 tons added at the ends to simulate the ballast tanks a more realistic value should be around $m_{\mathrm{eq}}=\sim 2150 \mathrm{~kg} / \mathrm{m}^{2}$. If this distributed mass is used, $\sqrt{ } \gamma=2.51$ and $f_{1}^{(0)}$ becomes $9.6 \mathrm{~Hz}$. However by considering the realistic model of the submarine with the conical end caps and the fluid loading with $m_{\mathrm{eq}}=\sim 2150 \mathrm{~kg} / \mathrm{m}^{2}$, the match of the frequencies is still achieved, as can be seen in Table 1. Therefore we will keep using the simplified model of a submarine in vacuum with $m_{\mathrm{eq}}=2896 \mathrm{~kg} / \mathrm{m}^{2}$ for a quick guide. It has been noted that the adjusting dimensional parameters of length $L$, radius $a$, hull 
TABLE 1: Comparison of the natural frequency of the simplified model structure $\left(m_{\mathrm{eq}}=2896 \mathrm{~kg} / \mathrm{m}^{2}\right)$ with the resonant frequency of the realistic model structure $\left(m_{\mathrm{eq}}=2150 \mathrm{~kg} / \mathrm{m}^{2}\right)$ for the $n=0$ mode and the $n=1$ mode vibrations. $L=113 \mathrm{~m}$. $(U, W)$ for $n=0$ and $(U, V, W)$ for $n=1$ are the normalized eigenvectors.

\begin{tabular}{ccc}
\hline$(n, m)$ & $\begin{array}{c}\text { Simplified model } \\
\text { Natural frequency }(\mathrm{Hz})(U, W)\end{array}$ & $\begin{array}{c}\text { Realistic model } \\
\text { Resonant frequency }(\mathrm{Hz})\end{array}$ \\
\hline$(0,1)$ & $8.5(0.999,0.022)$ & 8.6 \\
$(0,2)$ & $17.1(0.999,0.046)$ & 17.4 \\
$(0,3)$ & $25.5(0.997,0.046)$ & 25.7 \\
$(0,4)$ & $34.0(0.994,0.105)$ & 35.5 \\
\hline \multicolumn{3}{c}{$(U, V, W)$} \\
$(1,1)$ & $0.9(0.104,0.705,0.702)$ & 1.4 \\
$(1,2)$ & $3.3(0.175,0.701,0.691)$ & 3.5 \\
$(1,3)$ & $6.6(0.210,0.698,0.684)$ & 6.3 \\
$(1,4)$ & $10.4(0.219,0.696,0.684)$ & 9.3 \\
$(1,5)$ & $14.3(0.212,0.693,0.689)$ & 12.4 \\
$(1,6)$ & $18.2(0.197,0.686,0.700)$ & 14.7 \\
\hline
\end{tabular}

thickness $h$, distributed mass $m_{\mathrm{eq}}$, and so forth within $10 \%$ can still give harmonics of $8.5 \mathrm{~Hz}$ for the natural frequencies vibrations of the models. This result means the range of the uncertainty of the dimension of this model structure is less than $10 \%$. The solution of the second equation in (7) for the second branch of the eigenvalues gives under the condition of small $(\mathrm{ka})$ and small value of $\beta^{2} \sim 10^{-5}$

$$
\Omega^{2} \approx(1+\mu+2 \chi)
$$

Here $\mu \approx \chi=0.387$ and we get $f=81.6 \mathrm{~Hz}$. The accurate calculation of the corresponding frequencies gives $81.6 \mathrm{~Hz}$ $(m=1), 81.7 \mathrm{~Hz}(m=2), 81.8(m=3)$, and $85.0 \mathrm{~Hz}$. Since these frequencies are greater than the cut-off frequency $f_{c}=40 \mathrm{~Hz}$ of the seismic signals reported for this event, we will not consider this branch of frequencies any more.

2.3. Asymmetric Modes of Vibration. For the $n=1$ bending modes the solution of $\Omega^{2}$ in (4) gives three branches of eigenvalues and the corresponding three eigenvectors. The frequency for each $m$ can be calculated from the eigenvalues of $\Omega^{2}$. The lowest frequency branch represents mainly radial motion and is of interest here; the first 6 frequencies and the corresponding normalized eigenvectors are listed in Table 1. The corresponding resonant frequencies calculated with the realistic model are also listed in Table 1 . The frequencies of the second and the third branches of $\Omega^{2}$ are greater than the frequency range investigated and they will not be considered in this study. The vibrations with $n>1$ are out of consideration in this work because those give little contribution to the response as it will be shown later. It should be noted that in Table 1 the difference between the frequencies calculated using the simplified model with $m_{\mathrm{eq}}=2896 \mathrm{~kg} / \mathrm{m}^{2}$ and the realistic model with $m_{\mathrm{eq}}=2150 \mathrm{~kg} / \mathrm{m}^{2}$ is small supporting the usefulness of the simplified model.

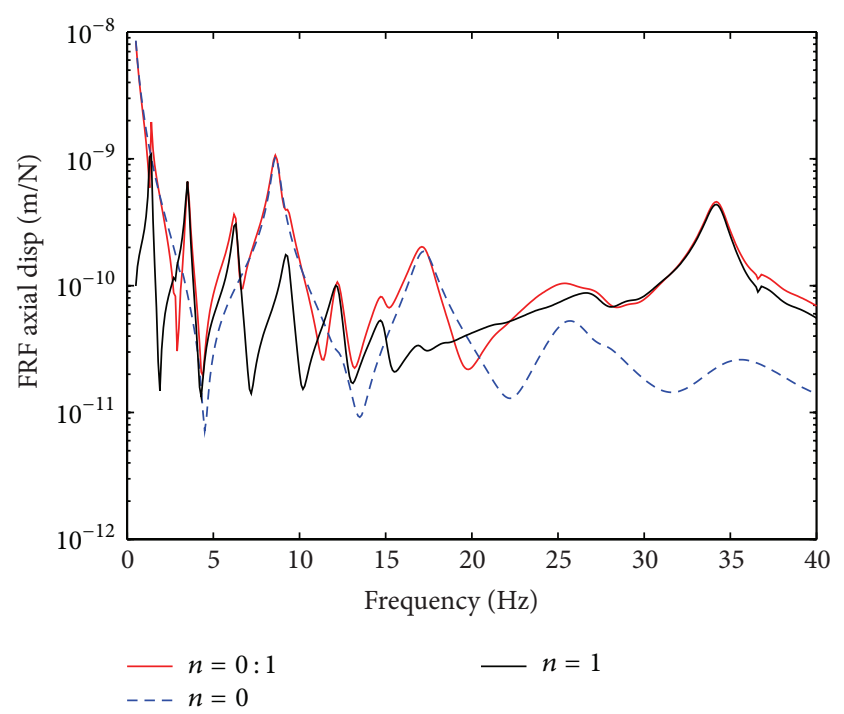

FIGURE 3: Frequency response function for an axial force applied to the submarine end.

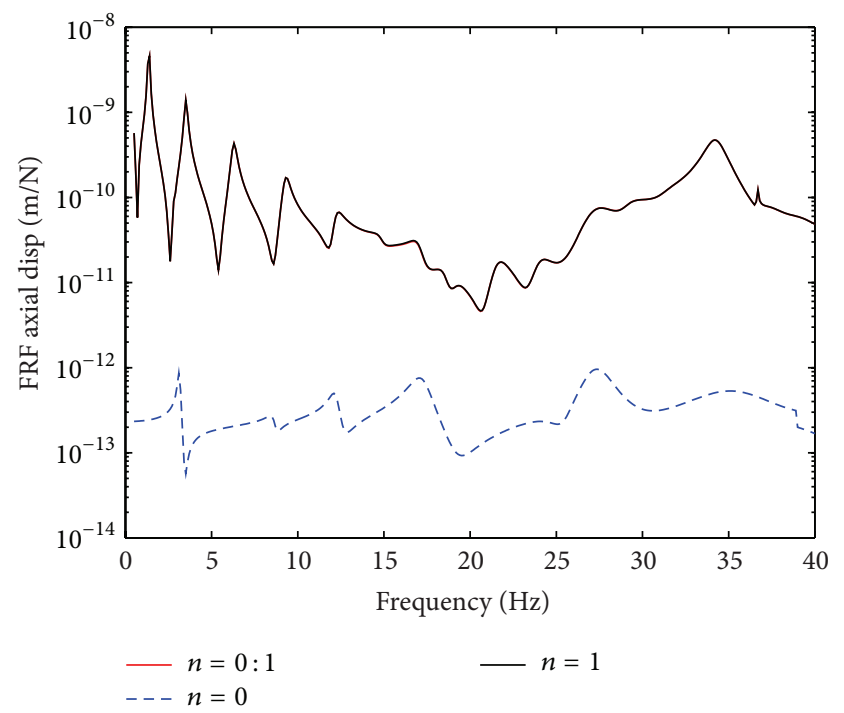

FIGURE 4: Frequency response function for a radial force applied to the submarine end.

\section{Structural and Acoustic Responses}

3.1. Structural Response. Figures 3 and 4 show the structural frequency response function (FRF) of the complete submarine model for the axial and radial displacement at the driving point, as applying a point unit force at one end of the cylindrical hull in axial direction. For the reciprocity principle the FRFs shown can be also interpreted as the response for the radial and axial displacement in response to a radial force applied at the same point. The calculations in these plots were carried out using the modeling approach presented in [12]. The contribution of the $n=0$ and $n=1$ modes is also shown in the same figure; modes with $n>$ 1 were excluded by the simulation since it did not give a significant contribution to the response. It has to be noted 
that those results are for a point force, in case the excitation force is well distributed around the hull, such as in the case of a front collision, the contribution on the response would be given only by the $n=0$ modes. The natural frequencies corresponding to the sharp peaks in the FRF are summarized in Table 1.

The striking feature in Figure 3 is the resonant frequencies of the $n=0$ mode, being around $8.6 \mathrm{~Hz}, 17.0 \mathrm{~Hz}, 25.0 \mathrm{~Hz}$, and $36.0 \mathrm{~Hz}$. The contribution of the $n=1$ modes is also important and an increase in response is observed at around $35.0 \mathrm{~Hz}$ where the waves of Class II (the second branch of W2) cut on, as it was also observed in [12]. These results show a qualitative similarity between the seismic signature and the natural frequencies of a large submarine, validating the possibility of a collision discussed before. The result also suggest a collision with the front part of the submarines with a main excitation of the axisymmetric $(n=0)$ modes of vibration. It can be seen in Figure 4 that the contribution of $n=0$ to the radial displacement is much lower than the axial displacements as shown in Figure 3. This aspect is consisting with the fact of low ratios of $W / U$ in the eigenvectors $(U, W)$ as in Table 1. Also it can be noted that the $n=1$ modes have a mainly radial displacement. This aspect can be expected by noting the ratios of $W / U$ in the eigenvectors $(U, V$, and $W)$ in Table 1.

It has to be mentioned that the purpose of this calculation is to show the FRF of the submarine model and its natural frequencies, and a possible qualitative correlation with the seismic data recorded. In reality the exact impact point on the hull, the actual force transmitted to the submarine, and its frequency content would surely affect the quantitative results but an exact reconstruction of the impact it is surely a huge and uncertain task and is out of the scopes of this work.

3.2. Radiated Sound at Far Field. The sound pressure radiated at far field was also calculated using the model in [12] and the corresponding wave displacement is shown in Figure 5 normalized as a displacement per unit force and per $\mathrm{km}$. The far field location is chosen to be in front of the submarine as a standard point for discussion. The trend of the figure is not much different from the seismic spectra reported in $[3,9]$ as discussed in Section 4. However this matching does not mean that the front of the submarine was toward to the BAR station, where Korea Meteorological Administration Station (37.9771N, 124.7142E) in Baekryong Island is [9] from where the seismic data analyzed in $[3,9]$ was obtained. The reason is that the waves from the vibration source of the conical shells of the submarine will propagate spherically in the far field through mediums. The path of this spherically propagated waves arrived at the BAR station was suggested in Figure 6 of [3] (see Figure 6 in this paper).

\section{Discussion}

Figure 7 of [3] showed the frequency spectrum of the pwave signals in $Z$-direction which was the Fourier transform of the first arrived p-waves at the BAR recording system in one second time window. Also Figure 3(b) of [9] showed the

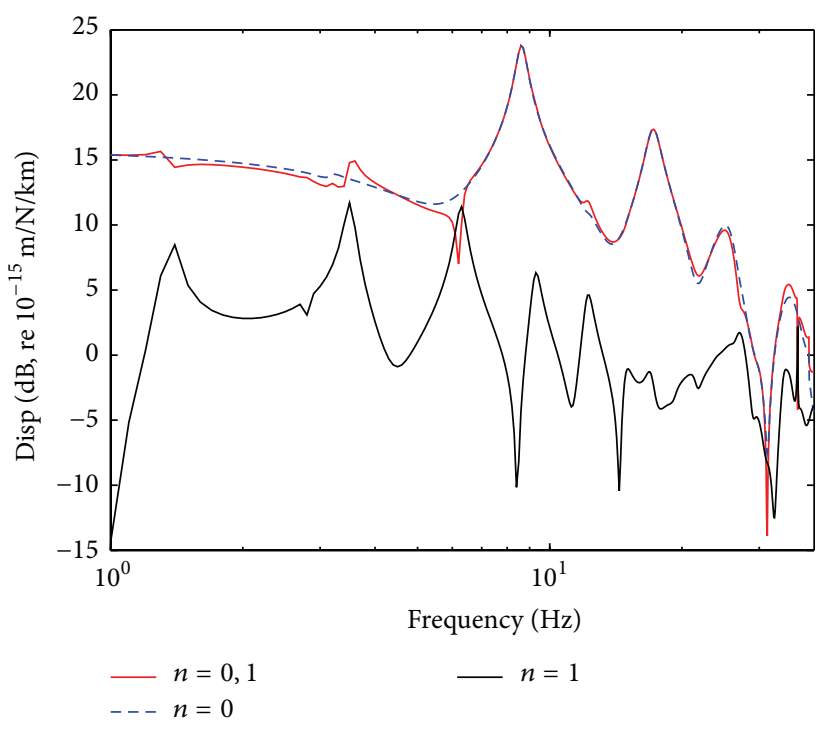

FIGURE 5: Far field wave displacement for axial force acting on the submarine end.

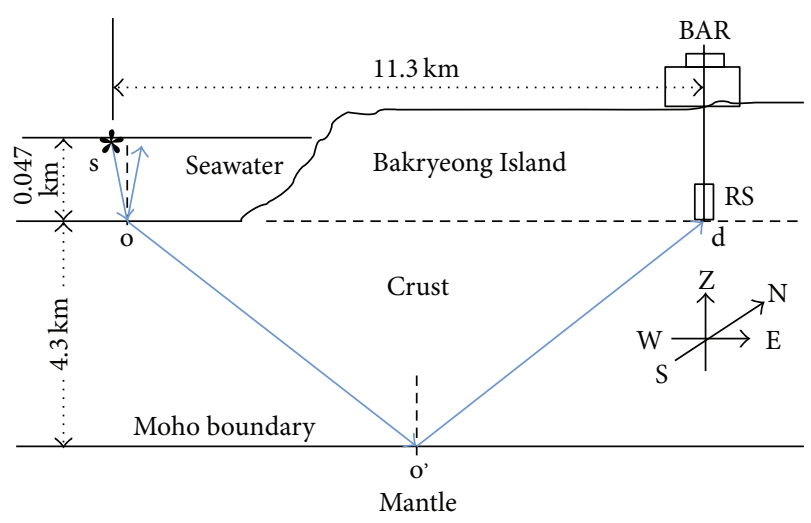

FIGURE 6: s: The source of the vibration of signals; RS: the recording system at BAR station. Table 4 of [3] lists that the sound velocity in sea water is $1.5 \mathrm{~km} / \mathrm{s}$; the velocities of $\mathrm{p}$-wave in crust and mantle are $6.3 \mathrm{~km} / \mathrm{s}$ and $7.95 \mathrm{~km} / \mathrm{s}$, respectively, while those of s-wave are $3.50 \mathrm{~km} / \mathrm{s}$ and $4.41 \mathrm{~km} / \mathrm{s}$. The densities of crust and mantle are $2.58 \mathrm{~kg} / \mathrm{m}^{3}$ and $3.30 \mathrm{~kg} / \mathrm{m}^{3}$, respectively.

normalized spectra of seismic signals in a time window of 15 seconds for each $Z$, EW (east-west direction) and NS (northsouth direction), separately using the same BAR recordings of the seismic signals. In this case the analyzed signals contain not only the first arrived p-wave, but the later arrived s-wave, Rayleigh, Love waves, and so forth. The Fourier transforms of all these waves gives the spectrum which is the sum of the spectrum of each signal travelled via several independent paths. As expected the spectrum in each direction of $Z, \mathrm{EW}$, and NS in Figure 3 of [9] showed similar patterns to each other. For the comparison of the spectrum in Figure 5 with these reported spectra, the data of the spectra peaks in Figure 7 of [3] and in Figure 3(b) of [9] are summarized in Tables 2 and 3, respectively. The frequencies of the peaks of Figure 5 are compared with the peaks of the reported spectra of 
TABLE 2: The amplitude data of the peaks in the frequency spectrum reported in Figure 7 of [3]. A-column is for meter unit scale, $B$ column is for nm unit, $C$-column is for the relative amplitudes divided by $8.5 \mathrm{~Hz}$ amplitude, and $D$-column is for the relative amplitudes of pressure converted from $C$-column displacement amplitudes.

\begin{tabular}{lccccc}
\hline$f(\mathrm{~Hz})$ & $A(\mathrm{~m})$ & $B(\mathrm{~nm})$ & $C$ & $D$ & Cal. $^{*}$ \\
\hline 8.5 & $10^{-8.22}$ & 6.0 & 1.0 & $1.0^{+}$ & 1.0 \\
17.7 & $10^{-8.88}$ & 1.3 & 0.22 & 0.45 & 0.23 \\
26.0 & $10^{-9.40}$ & 0.4 & 0.07 & 0.20 & 0.04 \\
34.6 & $10^{-10.17}$ & 0.07 & 0.01 & 0.05 & 0.016 \\
\hline
\end{tabular}

${ }^{+} 5.2 \mathrm{~Pa} .{ }^{*}$ Cal.: the estimated peak ratios from Figure 5, which are comparable with those in C-column.

Figure 7 of [3] in Table 2 and with the peaks of Figure 3(b) of [9] in Table 3.

In [9] the original unit scale was not specified in the spectra, but supposed to be in Pascal. Thus the difference between those in D column in Table 2 and those in the average in Table 3 may indicate uncertainty of amplitudes in the recorded seismic spectra. On the other hand, the peak ratios in Figure 5 are estimated to be 1.0 for $8.6 \mathrm{~Hz}, 0.23$ for $17.0 \mathrm{~Hz}, 0.04$ for $25.0 \mathrm{~Hz}$, and 0.016 for $36.0 \mathrm{~Hz}$ as listed in Cal.-column in Table 2. These ratios are close to those in Table 2: C-column, implying good fitting for both frequencies and amplitudes ratios too with the reported spectra. We also noted that the frequencies of natural vibrations of the hull frame of ROKS Cheonan were calculated and listed in 〈Table III-6-2〉 (p160) in [1], as $2.32 \mathrm{~Hz}, 4.74 \mathrm{~Hz}, 7.71 \mathrm{~Hz}$, $10.41 \mathrm{~Hz}$, and $13.40 \mathrm{~Hz}$ in the bending motion only. (No axial vibration is expected for the hull frame of the warship.) Clearly these natural frequencies could not be correlated with the characteristic harmonic frequencies $(8.5 \mathrm{~Hz}$ and the multiples) of the seismic signals recorded.

In summary the main peaks at $8.5 \mathrm{~Hz}$ and its multiples in the reported spectra of the seismic signals are reasonably consistent with the natural frequencies of the submarine with a length of around $113 \mathrm{~m}$. This result suggests a possibility of the collision between the Cheonan warship and the submarine. Then one might wonder about the damage that the submarine would have following a collision. We believe that the submarine would be negligibly damaged by the collision since the thickness of the hull of a large submarine is supposed to be more than $6 \mathrm{~cm}$ with and made of high strength steels, while the hull thickness of the ROKS Cheonan is known to be about $1.2 \mathrm{~cm}$ with steels and aluminum alloys in the upper parts. Besides we have found that it is no difficult with this collision theory discussed in this paper to illustrate damage aspects including deformations on the recovered bow and the stern of the split Cheonan warship from the sinking.

\section{Conclusion}

This study shows that the characteristic frequencies at spectral peaks of the seismic signals recorded at the time of the Cheonan incident are consistent with the natural frequencies of vibrations of a large submarine with a length
TABLE 3: The amplitude data of the peaks in the normalized frequency spectra for $Z$, EW, and NS seismic signals reported in Figure 3(b) of [9].

\begin{tabular}{lcccc}
\hline$f(\mathrm{~Hz})$ & $Z$ & EW & NS & Av. $^{*}$ \\
\hline 8.5 & 1.0 & 1.0 & 1.0 & 1.0 \\
17.0 & 0.30 & 0.38 & 0.22 & 0.30 \\
25.5 & 0.13 & 0.17 & 0.10 & 0.13 \\
34.0 & 0.03 & 0.03 & 0.02 & 0.03 \\
\hline
\end{tabular}

${ }^{*} \mathrm{Av}$. is for the average of peak amplitudes in Z, EW, and NS.

of around $113 \mathrm{~m}$. The matching is particularly good with the axisymmetric $(n=0)$ modes of vibrations suggesting a front collision with the submarine that might have caused the ROKS Cheonan sinking. The authors hope this work may be the starting point for new investigations to shine some light on the mysterious causes of the Cheonan sinking that caused the death of 46 people and for which a clear and definitive explanation has not been given yet.

\section{Appendices}

\section{A. Doubts about the JIG's Report}

Several doubts have been raised about the JIG's report and they are explained in what follows. The first issue of the controversy over the JIG's report [1] is on the validity of composition analysis results of the Al-containing white powder absorbed. These materials were found from the following three locations:

(A) the split ROKS Cheonan recovered in April of 2010;

(B) the remnants of a torpedo retrieved near the incident site on 15 May 2010;

(C) the explosion products from the small-scale explosion experiment using a highly aluminized explosive source of $15 \mathrm{~g}$ in a tank filled with 4.5 tons of seawater.

The composition analysis was performed with data obtained from SEM (scanning electron microscopy), EDS (energy dispersive spectrometer), and XRD (X-ray diffraction) for all three cases. The analysis was based on their knowledge or assumption: (1) XRD data of an amorphous aluminum oxide will not show any noticeable diffraction peaks. (2) When the aluminum is exposed to moisture, acids, and bases as in seawater environment for a long time, it forms naturally white corrosion products; the major components of these corrosion products are aluminum hydroxide $\left(\mathrm{Al}(\mathrm{OH})_{3}\right.$, bayerite) along with boehmite $(\mathrm{AlO}(\mathrm{OH}))$ and $\mathrm{Al}_{2} \mathrm{O}_{3}$, all of which are known to be crystalline, rather than amorphous. In the JIG analysis the EDS data of A, B, and C samples showed commonly prominent intensity peaks of oxygen and aluminum with ratio in $\mathrm{I}(\mathrm{O}): \mathrm{I}(\mathrm{Al})=0.9: 1$. JIG regarded this result as evidence that the absorbed materials of $\mathrm{A}, \mathrm{B}$, and $\mathrm{C}$ samples were aluminum oxides. The XRD data of the $\mathrm{A}$ and $\mathrm{B}$ samples did not show any noticeable X-ray diffraction peaks of an aluminum oxide crystal and the XRD data of $C$ sample showed prominent aluminum Bragg diffraction peaks 
and other weak peaks. These weak peaks were analyzed to be irrelevant to aluminum oxide crystals. However JIG did not give explicitly the reason why the strong Al Bragg peaks appeared in the XRD data and they thought that the XRD data of $\mathrm{C}$ sample also did not show any noticeable $\mathrm{X}$-ray diffraction peaks relevant to the aluminum oxide. These results led JIG to the conclusion that the $\mathrm{A}, \mathrm{B}$, and $\mathrm{C}$ samples were almost all $(\sim 100 \%)$ an amorphous aluminum oxide by (1). Because the product was an amorphous material, these materials could not be natural corrosion products of aluminum which are known to be crystalline by (2). Then JIG concluded that all these results were clear evidence that the adsorbed materials of $\mathrm{A}$ and $\mathrm{B}$ were amorphous aluminum oxides, which are the explosion product of the torpedo whose remnants were found near the incident site. We think the steps stated above were JIG's chain logic to draw the conclusion.

The second issue about JIG's report is whether the torpedo remnants were genuine or not. Inside the rear section of the torpedo remnants, JIG found Korean handwrite marking "1bun (No. 1 in English in blue ink)", similar to the marking of a North Korean test torpedo obtained in 2003, as seen in 〈Figure Summary-4〉 and 〈Figure Summary-5〉 of JIG's report. JIG declared promptly "we found conclusive evidence" for the cause of the incident. JIG said these evidences confirmed that the recovered torpedo parts were manufactured in North Korea and concluded that ROKS Cheonan was split and sunk due to shockwave and bubble effects generated by the underwater explosion of a torpedo, manufactured by North Korea. However in JIG's composition analysis data, Lee [4] indentified the XRD weak peaks for $\mathrm{C}$ sample, in fact, came from diffraction of a small amount of well crystallized $\alpha-\mathrm{Al}_{2} \mathrm{O}_{3}$, contrary to JIG's claim that the weak peaks were irrelevant to aluminum oxide crystals. One can see clearly weak $\alpha-\mathrm{Al}_{2} \mathrm{O}_{3}$ Bragg diffraction peaks in $\langle$ Figure Appendix v-5-3〉, p280 in JIG's report [1]. We think this finding broke JIG's chain logic.

This result means that at least the $\mathrm{C}$ sample was quite different from the $\mathrm{A}$ and $\mathrm{B}$ samples, and in turn JIG's conclusion above is very questionable, as Lee has concluded. Lee and Yang then reported their own experimental results in another study [5]. They presented the XRD data in Figure 2 of [5] for the sample of an Al-powder that underwent melting followed by rapid quenching. This figure showed prominent $\mathrm{Al}$ Bragg diffraction peaks and weak $\alpha-\mathrm{Al}_{2} \mathrm{O}_{3}$ Bragg diffraction peaks. They said that "this clearly indicates Al-powder oxides partially, not entirely, during the heating and rapid quenching." This oxidation was supposed to occur on the surface thin layers of each grain of Al powder. The XRD data in Figure 2 of [5] showed the similar pattern as in $\langle$ Figure Appendix V-5-3> of JIG's report for C sample. Nevertheless the EDS data in Figure 1 of [5] shows markedly different from $\langle$ Figure Appendix V-5-2〉 of JIG's report for C sample. That is, the peak ratio of $\mathrm{I}(\mathrm{O}) / \mathrm{I}(\mathrm{Al})$ in Figure 1 of [5] was measured as 0.25 , whereas $\langle$ Figure Appendix v-5-2 $\rangle$ showed very different values of 0.81 . On the other hand, the authors in [5] noticed that the high value of around $0.8-0.9$ for $\mathrm{I}(\mathrm{O}) / \mathrm{I}(\mathrm{Al})$ in EDS data was expected for aluminum hydroxide, such as $\mathrm{Al}(\mathrm{OH})_{3}$. They also showed the simulation results of $\mathrm{Al}(\mathrm{OH})_{3}$ and $\mathrm{Al}_{2} \mathrm{O}_{3}$ in Figure 4 of [5], supporting their expectation. (It should be noted that the EDS intensity data comes from the surface thin layers of the sample. That is, the EDS data of Figure 1 of [5] came from the surface thin layers of oxidized grains with $\alpha-\mathrm{Al}_{2} \mathrm{O}_{3}$ of the heat treated Al-powder sample.) From these results they concluded that JIG's adsorbed materials taken from the warship and the torpedo remnants were not associated with any explosion and that JIG's EDS data of their test-explosion sample (Figure Appendix v-52) were likely fabricated. The South Korean government has adamantly denied any fabrication or any major problems with its interpretation of the data. Later on, independently the $\mathrm{A}$ and $\mathrm{B}$ samples were analyzed to be a basaluminite $\left[\mathrm{Al}_{4}\left(\mathrm{SO}_{4}\right)(\mathrm{OH})_{10} \cdot 4-5 \mathrm{H}_{2} \mathrm{O}\right]$ materials by Professor Jeong GY in Earth \& Environmental Science from Andong National University in Korea, using several instruments including a TEM. He said that the materials appeared to be formed for a long time such as in seawater, implying they had nothing to do with explosions. Unfortunately he was not able to test the C sample.

Cyranoski [6] reported in Nature online the summary of the controversy over JIG's report. Some important points raised in the article are as follows.

(a) An expert investigator was placed on the JIG by the opposition party-Shin Sang-chul, a former officer in the South Korean navy who had also worked at a shipbuilding company-suggested, before the report was even released, that an accidental collision with a US warship, and not North Korea, was to blame. The United States and South Korea had been carrying out military exercises in the area at the time. Now he is insisting on the collision with a submarine with a length of around $60 \mathrm{~m}$, not by a US warship.

(b) The report's claim that a torpedo-induced water column sank the Cheonan contradicted earlier testimony from survivors that they did not see that water column. They also testified neither seeing any explosion flash from the underwater nor smelling of explosives at the time of the incident.

Lee and Suh in [7] reported in Policy Forum 10-039 "Inconsistencies in South Korea's Cheonan Report." In the article we found particularly interesting the following point.

"If the bottom of the ship was hit by a bubble, it should show a spherical concave deformation resembling the shape of a bubble, as the JIG's own simulation suggests in the Appendix of the report, but it does not. The bottom of the front part of the ship is pushed up in an angular shape, as the yellow line shows in 〈Figure III-1-7〉 of the report, more consistent with a collision with a hard object. Suh has further argued that, contrary to common reports that the Cheonan was split in half as also seen in JIG's simulation, it actually broke into two larger pieces as well as a third smaller piece (see Figure 1 in this paper)." Shin Sang-chul clearly pointed out if the warship was split by a bubble jet generated from the non-contact underwater explosion, the top hull of the recovered broken warship should show upward bending, but it does not seem the case as can be seen in 〈Figure II-3-3 and $4\rangle$ and $\langle$ Figure III-1-8 $\rangle$ of the report. 
Kim et al. in "Foreign Policy In Focus" [8] reported briefly skeptics on JIG's judgment about the Korean handwrite marking "1bun" on the rear section of the torpedo remnants. JIG claimed this handwriting marking had been written by a North Korean in the process of manufacturing the torpedo and is the conclusive evidence that the torpedo was made in North Korea. Nevertheless Shin Sang-chul found the trace that the rusts on the panel of the rear section of the torpedo remnants had been removed by a sand-paper; after the rust was removed the marking of Korean words "1bun" was written on the panel. He said, therefore, this marking could not be "conclusive evidence" that the torpedo was made in North Korea. One can judge whether his judgment is correct or not from 〈Figure Summary-4〉 of the report. We personally think Shin Sang-chul is right. It is reminded that the remnants were fragments following the torpedo explosion and had been deposited in the mud of seabed for 50 days and recovered by the special fishing net [1]. Considering all the several doubts explained above we believe the torpedo remnants they found near the incident site could not be a genuine conclusive evidence for the cause of the incident. Furthermore the conclusion was drawn after only five days of work (the remnant was found on 15 May 2010 and JIG's report of a summary of its investigation was released on 20 May 2010).

\section{B. The Free Vibrations of a Cylindrical Shell with Shear-Diaphragm Boundary Conditions}

The Flügge equations of motion for the vibrations of a ring stiffened cylindrical shell are given by Caresta and Kessissoglou [12]:

$$
\begin{gathered}
\frac{\partial^{2} u}{\partial z^{2}}+\frac{(1-v)}{2 a^{2}}\left(1+\beta^{2}\right) \frac{\partial^{2} u}{\partial \theta^{2}}+\frac{(1+v)}{2 a} \frac{\partial^{2} v}{\partial z \partial \theta} \\
+\frac{v}{a} \frac{\partial w}{\partial z}-\beta^{2} a \frac{\partial^{3} w}{\partial z^{3}}+\beta^{2} \frac{(1-v)}{2 a} \frac{\partial^{3} w}{\partial z \partial \theta^{2}}-\frac{\gamma}{c_{L}^{2}} \frac{\partial^{2} u}{\partial t^{2}}=0 \\
\frac{(1+v)}{2 a} \frac{\partial^{2} u}{\partial z \partial \theta}+\frac{(1-v)}{2} \frac{\partial^{2} v}{\partial z^{2}}+\frac{1+\mu}{a^{2}} \frac{\partial^{2} v}{\partial \theta^{2}} \\
+\frac{1+\mu+\chi}{a^{2}} \frac{\partial w}{\partial \theta}+\frac{\chi}{a^{2}} \frac{\partial^{3} w}{\partial \theta^{3}} \\
+\beta^{2}\left(\frac{3(1-v)}{2} \frac{\partial^{2} v}{\partial z^{2}}-\frac{3-v}{2} \frac{\partial^{3} w}{\partial z^{2} \partial \theta}\right)-\frac{\gamma}{c_{L}^{2}} \frac{\partial^{2} v}{\partial t^{2}}=0
\end{gathered}
$$$$
\beta^{2}\left(a^{2} \frac{\partial^{4} w}{\partial z^{4}}+2 \frac{\partial^{4} w}{\partial z^{2} \partial \theta^{2}}+\frac{1}{a^{2}} \frac{\partial^{4} w}{\partial \theta^{4}}-a \frac{\partial^{3} u}{\partial z^{3}}\right.
$$$$
\left.+\frac{1-v}{2 a} \frac{\partial^{3} u}{\partial z \partial \theta^{2}}-\frac{3-v}{2} \frac{\partial^{3} v}{\partial z^{2} \partial \theta}+\frac{2}{a^{2}} \frac{\partial^{2} w}{\partial \theta^{2}}\right)
$$

$$
\begin{aligned}
& +\frac{v}{a} \frac{\partial u}{\partial z}+\frac{\chi}{a^{2}} \frac{\partial^{3} v}{\partial \theta^{3}}+\frac{2 \chi}{a^{2}} \frac{\partial^{2} w}{\partial \theta^{2}}+\frac{1+\mu+\chi}{a^{2}} \frac{\partial v}{\partial \theta} \\
& +\frac{1+\mu+2 \chi}{a^{2}} w+\frac{\gamma}{c_{L}^{2}} \frac{\partial^{2} w}{\partial t^{2}}=0 .
\end{aligned}
$$

Here $u, v$, and $w$ are the orthogonal components of shell displacements in the $z$-axial, the $\theta$-circumferential, and the $r$ radial directions in time $t$, respectively. The meanings of the symbols in the equations are defined in Section 2. The general solutions for shear diaphragm boundary conditions can be written as in [13]:

$$
\begin{gathered}
u(z, \theta, t)=U \cos (n \theta) \cos \left(k_{n} z\right) e^{-j w t} \\
v(z, \theta, t)=V \sin (n \theta) \sin \left(k_{n} z\right) e^{-j w t} \\
w(z, \theta, t)=W \cos (n \theta) \sin \left(k_{n} z\right) e^{-j w t} .
\end{gathered}
$$

These solutions represent standing waves in both the axial and circumferential directions, with $n$ nodal lines in the $\theta$ direction and with nodal cross-sections spaced at a distance $2 \pi / k_{n}$ in the $z$ direction. $k_{n}$ is the axial wave number and $n$ is the circumferential mode number. $j$ is the imaginary unit. The shear diaphragm boundary conditions at $z=0, L$, are given by

$$
\begin{gathered}
N_{z}=\frac{E h}{1-v^{2}}\left(\varepsilon_{z}+v \varepsilon_{\theta}+\frac{h^{2}}{12 a} K_{z}\right)=0 \\
M_{z}=\frac{E h^{3}}{12\left(1-v^{2}\right)}\left(\frac{\varepsilon_{z}}{a}+v \varepsilon_{\theta}+K_{z}\right)=0 \\
v(z, \theta, t)=0 \\
w(z, \theta, t)=0 .
\end{gathered}
$$

The membrane force $N_{z}$ and the bending moment $M_{z}$ are expressed as functions of the normal strains in the middle surface, $\varepsilon_{z}$ and $\varepsilon_{\theta}$, as well as the mid-surface changes in curvature, $K_{z}, K_{\theta}$. The expressions for the strain and changes in curvature are given by Leissa [13] as

$$
\begin{gathered}
\varepsilon_{z}=\frac{\partial u}{\partial z} \\
\varepsilon_{\theta}=\frac{1}{a}\left(\frac{\partial v}{\partial \theta}+w\right), \\
K_{z}=-\frac{\partial^{2} w}{\partial z^{2}}, \\
K_{\theta}=\frac{1}{a^{2}}\left(\frac{\partial v}{\partial \theta}-\frac{\partial^{2} w}{\partial \theta^{2}}\right) .
\end{gathered}
$$

As explained in [13], the shear diaphragm boundary conditions in (B.5) to (B.8) can be justified for a rigid thin, circular cover plate at each end, so that $v$ and $w$ displacements are restrained at both end boundaries. However the plates, by virtue of their thinness, would have very little stiffness in 
the $z$ direction transverse to their planes; consequently, they would generate negligible bending moment $M_{z}$ and longitudinal membrane force $N_{z}$ in the shell as the shell deforms. Under these boundary conditions, the axial wave number becomes $k_{n}=m \pi / L$, where $m=0,1,2, \ldots$ is the axial mode number. The natural frequencies of the shell can be found substituting the solution (B.4) with $k_{n}=m \pi / L$ into (B.3) and arrange in matrix form. For a nontrivial solution the determinant of the matrix must be zero. Expansion of the determinant leads to the characteristic equation of the shell. In vacuum, the dispersion equation is of sixth order in $\omega$. From this equation three different natural frequencies can found for each value of $k_{n}$.

B.1. Frequency Response Function (FRF). Suppose that the shell is axially excited at one end by a point force of unity amplitude located at $\left(z_{0}, \theta_{0}\right)$. The force can be described in terms of a Dirac delta function in function of the arc length $\sigma=a \theta$

$$
F\left(z_{0}, \theta_{0}, t\right)=F_{0} \delta\left(\sigma-\sigma_{0}\right) .
$$

The equilibrium of the membrane force, $N_{z}$, given by (B.5) evaluated at $z=z_{0}$ becomes

$$
\left.\frac{E h}{1-v^{2}}\left(\varepsilon_{z}+v \varepsilon_{\theta}+\frac{h^{2}}{12 a} K_{z}\right)\right|_{z=z_{0}}=F_{0} \delta\left(\sigma-\sigma_{0}\right) .
$$

The delta function $\delta\left(\sigma-\sigma_{0}\right)$ can be expanded as a Fourier series around the circumference as

$$
\delta\left(\sigma-\sigma_{0}\right)=\frac{1}{T_{z 0}}+\frac{2}{T_{z 0}} \sum_{n=1}^{\infty} \cos \left(\frac{2 \pi n \sigma}{T_{z 0}}\right) .
$$

With $T_{z 0}=2 \pi a$, (B.11) can be rewritten as

$$
\begin{aligned}
\frac{E h}{1-v^{2}} & \left.\left(\varepsilon_{z}+v \varepsilon_{\theta}+\frac{h^{2}}{12 a} K_{z}\right)\right|_{z=z_{0}} \\
= & \frac{1}{T_{z 0}}+\frac{2}{T_{z 0}} \sum_{n=1}^{\infty} \cos \left(\frac{2 \pi n \sigma}{T_{z 0}}\right) .
\end{aligned}
$$

For every circumferential mode number $n$, the 8 boundary equations, as expressed in [12] for the 3 membrane force, 3 bending moments, transverse shearing, and the KelvinKirchoff shear force-together with the equilibrium of the forces under point force excitation, can be arranged in the matrix form $\mathbf{A x}=\mathbf{F}$. $\mathbf{x}$ is the vector of the 8 unknown displacement coefficients and $\mathbf{F}$ is an $8 \times 1$ force vector with only one nonzero term $\varepsilon F_{0}$, where $\varepsilon=1 / 2 \pi a$ if $n=0$ and $\varepsilon=1 / \pi a$ if $n \neq 0$. Structural damping can be introduced using a complex Young modulus $E=E(1-j \eta)$ where $\eta$ (about 0.02) is the structural loss factor. Solving the system for each circumferential mode number gives the steady state shell displacement response at a certain frequency (FRF).

\section{The Path of the Signals Arriving at the BAR Recording System}

The optimized path of the signals arriving at the BAR station from the submarine is drawn in Figure 6. In this figure the waves having the velocity $1.5 \mathrm{~km} / \mathrm{s}$ in seawater with the incident angle $10.9^{\circ}$ at $\mathrm{o}$ on the boundary of the seabed/crust, refracted into the crust with the angle $52.4^{\circ}$ in case of p-waves (the velocity $6.3 \mathrm{~km} / \mathrm{s}$ in crust) by Snell's law. Under this condition the refracted waves will have maximum amplitudes with the transmission coefficient 0.174 . Then the waves reflected on the Moho boundary should continuously travel to the BAR recording system. This path is drawn as o-o' $-\mathrm{d}$ in Figure 6. For the case of s-waves with velocity of $3.5 \mathrm{~km} / \mathrm{s}$ in crust, the incident angle on the boundary must be $19.9^{\circ}$ to have the same path of $\mathrm{o}-\mathrm{o}^{\prime}-\mathrm{d}$. For all $\mathrm{p}$ and s-waves the refraction angle is $90^{\circ}$ at $\mathrm{o}^{\prime}$ with the zero transmission coefficients. To have this optimized o-o' $-\mathrm{d}$ with $14.22 \mathrm{~km}$ distance, the depth of the Moho boundary must be $\sim 4.3 \mathrm{~km}$. It is generally known that the depth of Moho boundary under sea is usually $5 \sim 7 \mathrm{~km}$. As considering this fact, we think the estimated depth of the Moho boundary about $4.3 \mathrm{~km}$ is acceptable in this case.

\section{Conflict of Interests}

The authors declare that there is no conflict of interests regarding the publication of this paper.

\section{References}

[1] JIG (Multinational Civilian-Military Joint Investigation Group), Joint Investigation Report: On the Attack against ROKS Cheonan, Military of National Defenses, ROK, 2010, http://cheonan46.go.kr/100.

[2] ROKS Cheonan sinking, http://en.wikipedia.org/wiki/ROKS_ Cheonan_sinking.

[3] T.-K. Hong, "Seismic investigation of the 26 March 2010 sinking of the South Korean naval vessel Cheonanham," Bulletin of the Seismological Society of America, vol. 101, no. 4, pp. 1554-1562, 2011.

[4] S.-H. Lee, "Comments on the section "adsorbed material analysis" of the Cheonan report made by the South Korean civil and military joint investigation group (CIV-MIL JIG)," http://arxiv.org/abs/1006.0680v2.

[5] S.-H. Lee and P. S. Yang, "Was the "Critical Evidence" presented in the South Korean Official Cheonan Report fabricated?" http://arxiv.org/abs/1006.0680v4.

[6] D. Cyranoski, "Controversy over South Korea's sunken ship," Nature, July 2010, http://www.nature.com/news/2010/100708/ full/news.2010.343.html.

[7] S.-H. Lee and J. J. Suh, "Rush to judgment: inconsistencies in South Korea's Cheonan report," Policy Forum 10-039, The AsiaPacific Journal: Japan Focus, 2010, http://www.japanfocus.org/ -Seunghun-Lee/3382.

[8] H.-E. Kim, G. Chaffin, and P. Certo, The Cheonan Incident: Skepticism Abounds, Foreign Policy in Focus, Washington, DC, USA, 2010.

[9] S. G. Kim and Y. Gitterman, "Underwater explosion (UWE) analysis of the ROKS cheonan incident," Pure and Applied Geophysics, vol. 170, no. 4, pp. 547-560, 2013.

[10] M. S. Weinstein, "Spectra of acoustic and seismic signals generated by underwater explosions during Chase experiment," Journal of Geophysical Research, vol. 73, pp. 5473-5476, 1968. 
[11] M. Caresta, Structual and acoustic responses of a submerged vessel [Ph. D thesis], Mechanical Engineering, University of New South Wales, Sydney, Australia, 2009.

[12] M. Caresta and N. J. Kessissoglou, "Acoustic signature of a submarine hull under harmonic excitation," Applied Acoustics, vol. 71, no. 1, pp. 17-31, 2010.

[13] A. W. Leissa, Vibration of Shells, American Institute of Physics, New York, NY, USA, 1993. 

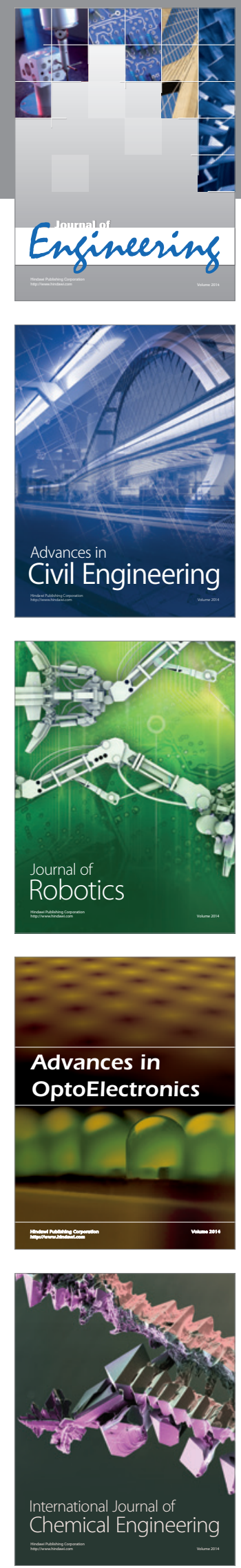

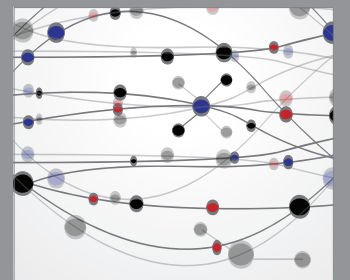

The Scientific World Journal
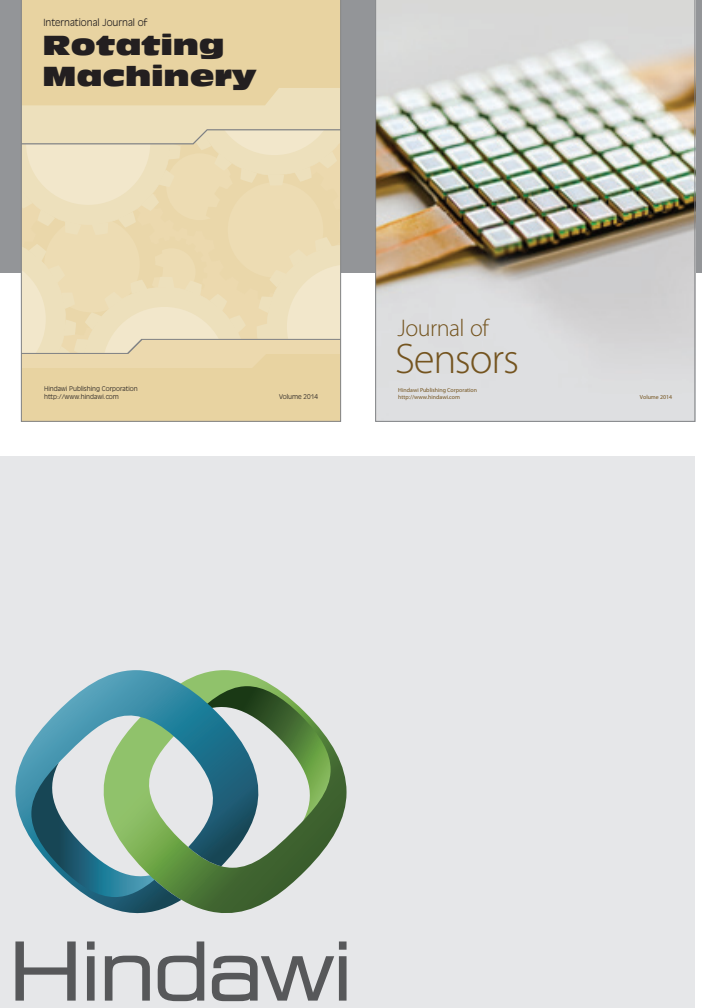

Submit your manuscripts at http://www.hindawi.com
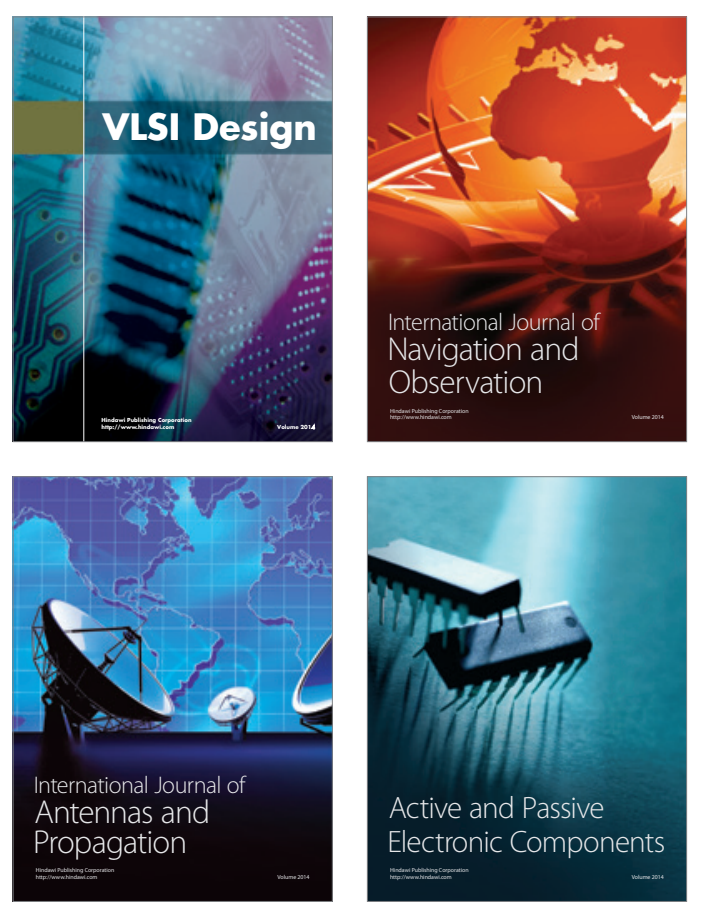
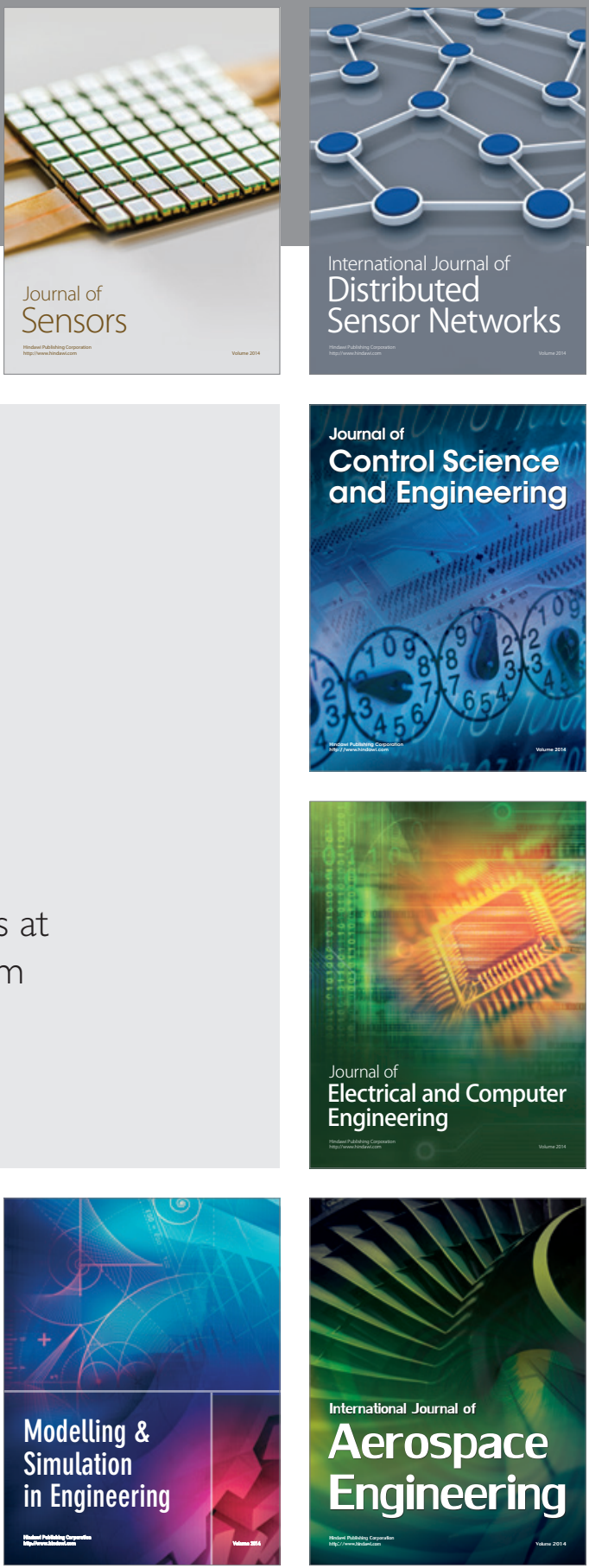

Journal of

Control Science

and Engineering
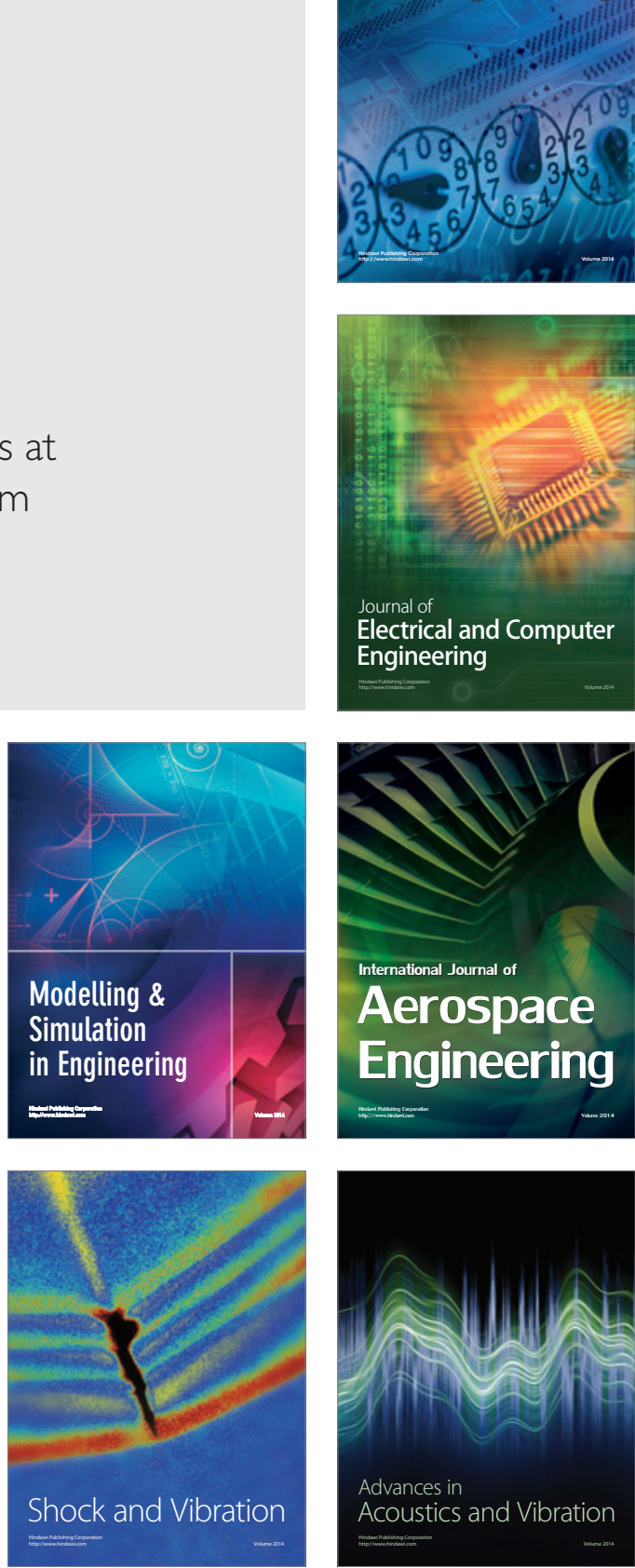